\title{
A Novel IGC Scheme for RHV with the Capabilities of Online Aerodynamic Coefficient Estimation and Trajectory Generation
}

\author{
Xueyun Wang ${ }^{1,2, *}$, Yifan $\mathrm{Li}^{1}$ and Jingjuan Zhang ${ }^{1,2}$ \\ 1 School of Instrument Science and Opto-electronics Engineering, Beihang University, Beijing 100191, China; \\ liyifann@buaa.edu.cn (Y.L.); zhangjingjuan@buaa.edu.cn (J.Z.) \\ 2 Hefei Innovation Research Institute, Beihang University, Hefei 230012, China \\ * Correspondence: wangxueyun@buaa.edu.cn
}

Citation: Wang, X.; Li, Y.; Zhang, J. A Novel IGC Scheme for RHV with the Capabilities of Online Aerodynamic Coefficient Estimation and Trajectory Generation.

Mathematics 2021, 9, 172.

https://doi.org/10.3390/ math9020172

Received: 18 December 2020

Accepted: 13 January 2021

Published: 15 January 2021

Publisher's Note: MDPI stays neutral with regard to jurisdictional claims in published maps and institutional affiliations.

Copyright: (c) 2021 by the authors. Licensee MDPI, Basel, Switzerland. This article is an open access article distributed under the terms and conditions of the Creative Commons Attribution (CC BY) license (https:/ / creativecommons.org/licenses/by/ $4.0 /)$.

\begin{abstract}
A novel integrated guidance and control (IGC) scheme for a Re-entry Hypersonic Vehicle (RHV) is proposed with the capabilities of online aerodynamic coefficient estimation based on an Unscented Kalman Filter and online trajectory generation based on the Gaussian pseudospectral method. A linear quadratic regulator is adopted for trajectory tracking guidance and a second-layer sliding mode controller is designed for attitude control. The variation of lift and drag coefficients are modeled and estimated online, based on which a new trajectory can be generated. The commands of trajectory generation are set as moments of actuators and their extremums pose more constraints on the rate and acceleration of flow angles. Comprehensive simulations are conducted and comparable IGC performances with normal conditions are obtained under large aerodynamic coefficient errors according to online generated trajectory, which proves the effectiveness and advantages of the proposed IGC scheme.
\end{abstract}

Keywords: integrated guidance and control; re-entry hypersonic vehicle; online trajectory generation; aerodynamic coefficient estimation

\section{Introduction}

When a re-entry hypersonic vehicle is launched, it reaches a specific altitude and then re-enters the atmosphere from near-Earth orbit. The lifting body configuration is favored for re-entry hypersonic vehicles (RHVs) due to the advantages of high maneuverability and great longitudinal and lateral ranges [1,2]. There are many cutting edge techniques for RHVs and one of them is guidance, navigation and control (GNC). Authors have conducted research on high-accuracy integrated navigation technologies for RHVs [3,4] and this paper focuses on integrated guidance and control. The mathematical foundation of re-entry guidance and control is the optimal solution for tightly coupled, high-rank nonlinear problems with complicated constraints under disturbances and uncertainties. Therefore, the design of guidance and control system for RHVs is a multiobjective, highly constrained and strongly nonlinear control problem. For lifting body RHVs, the states of the guidance and control nonlinear systems include position, velocity, attitude and flow angles while the actuators (commands) include moments imposed on the RHV through aerodynamic forces. For re-entry flight, the guidance and control are tightly coupled and their mutual influences are non-negligible, so integrated guidance and control (IGC) is necessary. Additionally, during re-entry the IGC systems are challenged by many uncertainties such as high-altitude wind speeds up to $170 \mathrm{~m} / \mathrm{s}$ [1], mathematical model errors under large angle of attack (AOA) and even insufficient failure of actuators. Under these scenarios, inaccuracy of aerodynamic coefficients may happen. Additionally, in cases of actuator failure and target change, a new trajectory needs to be generated online for guidance and control. Capabilities of online trajectory generation and online coefficient estimation will improve the IGC performance of RHVs, widening their mission range and reinforcing reliability. Research has been conducted related to IGC, trajectory generation and aerodynamic coefficient estimation. 
The Gauss pseudospectral method (GPM) is widely used for trajectory generation [5-7]. A novel time-optimal memetic whale optimization algorithm integrating the GPM is proposed in [5] for the hypersonic vehicle re-entry trajectory optimization problem with no-fly zones, proving that the GPM possesses a rapid convergence speed around the optimum and higher accuracy in the trajectory construction field. Mao et al. also applied the GPM for re-entry trajectory optimization because of its high efficiency and accuracy [6]. The GPM is also adopted to generate trajectory offline for guidance and control systems of re-entry vehicles in the presence of control constraints and multiple disturbances based on unified enhanced trajectory linearization control (TLC) [7]. Other methods of trajectory generation are studied [8-10] but the solutions are suboptimal due to online application. Generally, in the above research on GPM, the trajectory is generated with commands of flow angles-namely, AOA, bank angle (BA) and sideslip angle. However, the real commands of RHV guidance are moments and forces provided by control surfaces or a Reaction Control System (RCS). The constraints of angular rates and accelerations of the flow angles should also be considered along with the magnitude of the flow angles when generating the trajectory, otherwise the required flow angles of commands may not be accessible.

Various IGC schemes have been proposed by researchers. An IGC scheme is developed for a reusable launch vehicle (RLV) in [11] with the aim of improving the flexibility, safety and autonomy. An outer loop re-entry guidance law with online trajectory reshaping capability is designed and a novel re-entry attitude control strategy is proposed based on a sliding mode controller (SMC) and disturbance observer. A three-dimensional partial IGC law is proposed in [12] for cooperative flight of multiple hypersonic re-entry missiles. An IGC scheme combining with the virtual target and the filter for fixed-trim RHV is proposed in [13]. The IGC is based on a back-stepping method and an Extended State Observer (ESO) is designed for estimating the acceleration of the virtual target. Additionally, a small-gain method for IGC in the terminal phase of re-entry is designed using an SMC [14]. In order to reduce control chattering, the virtual control is introduced into the design of third-order SMC [15]. Shao et al. propose a novel composite IGC law for a missile intercepting against an unknown maneuvering target with multiple uncertainties based on the back-stepping technique. [16]. More research on guidance and control is detailed in [17-19]. It can be summarized that many efforts have been made to improve the capacity of resisting disturbances and overcoming coefficient uncertainty in the field of re-entry IGC.

In the field of online coefficient estimation, Seo et al. developed two Kalman filter based techniques to estimate aerodynamic coefficients for fixed-wing aircraft in upset conditions [20]. The proposed methods are tested with real ordinary flight data sets but the re-entry scenario is not discussed. Additionally, Ferreres et al. conducted research on the adaptive Linear Fractional Transformation (LFT) control of a civil aircraft with online frequency-domain parameter estimation [21] and Subrahmanyam et al. also developed an online identification system in the frequency domain for mini cropped delta Unmanned Aerial Vehicles (UAVs) [22]. Chowdhary et al. compare the performance of three online parameter estimation algorithms with real flight data from two aircrafts-a fixed-wing aircraft (HFB-320) and a rotary wing UAV (ARTIS). An Extended Kalman Filter (EKF) and Unscented Kalman Filter (UKF) are compared and results show that the UKF performs better [23]. Similarly to all the work above, only aircrafts in normal conditions are researched, showing a lack of high-maneuver and large AOA scenarios of re-entry flight. A novel robust adaptive gliding guidance strategy based on online identification of aerodynamic coefficients for hypersonic vehicles is proposed in [24]. Aerodynamic models are constructed in the form of quadratic polynomial functions and an Extended Kalman Filter (EKF) is employed to estimate the unknown parameters. Additionally, online aerodynamic coefficient identification based on EKF is tested with real data from two hypersonic vehicles, X-31 and HARV [25], showing the effectiveness of coefficient estimation. However, considering the one-order approximation accuracy of EKF, it can be improved by applying UKF whose approximation accuracy is at least second-order. Additionally, the computation 
complexity is relieved for online applications in the case of UKFs since a Jacobian matrix is not necessary.

In the case of RHV actuator failure, the control capability is crippled, leading to the original target or the landing site not being reachable. Firstly, the changed aerodynamic coefficients need to be estimated online for trajectory regeneration and IGC. Secondly, a new trajectory needs to be generated online to guide the RHV to a feasible landing site. Additionally, online trajectory generation is also required for a changing target scenario; online aerodynamic coefficient estimation is also meaningful in terms of improving the IGC performance under obvious disturbances and uncertainty.

In this paper, a novel IGC scheme for RHV is proposed with the capabilities of online aerodynamic coefficient estimation based on UKF and online trajectory generation based on the GPM. A Linear Quadratic Regulator (LQR) is adopted for trajectory tracking guidance and a second-layer SMC is designed for attitude control. The variation of lift and drag coefficients are modeled and estimated online through a UKF. In the procedure of online trajectory generation, commands are set as moments of actuators and their extremums impose constraints on the rate and acceleration of flow angles such as AOA and BA. Comprehensive simulations are conducted to prove the effectiveness and advantages of the proposed IGC scheme.

Compared with other known re-entry IGC schemes, the following contributions are made by our research from a scientific view: (1) Constraints of the rate and acceleration of flow angles are considered in trajectory generation based on the GPM. (2) Dynamic models with 6 degrees of freedom (DOFs) are applied in the guidance laws of LQR, which takes the RHV's control constraints into account and can provide expected flow angles and their rates for attitude control system. (3) During guidance lift and drag, coefficients of RHV are estimated online for re-entry vehicles through UKF for robust IGC and possible online trajectory construction.

The remainder of this paper is organized as follows. In Section 2, the dynamics models and multi-constraints during re-entry are presented. Section 3 introduces the novel IGC scheme, including the online trajectory generation algorithm and coefficient estimation algorithm. The simulations are shown in Section 4 and conclusions are drawn in Section 5.

\section{Problem Formulation}

The dynamic models and multiple constraints for unpowered lifting re-entry hypersonic vehicles are described in this section.

\subsection{Equations of Motion}

Several assumptions are made to derive the aerodynamic mathematical model:

(1) The Earth is a spherical rotating well-distributed planet;

(2) The mass of the RHV is constant;

(3) The RHV is rigid;

(4) The RHV is a bank-to-turn (BTT) aircraft and side force is negligible;

(5) The RHV has axial symmetry and moments of inertia of crossed axes are negligible.

On these assumptions, the three degrees of freedom (DOFs) point-mass dynamics of the unpowered lifting RHV is described by the following equations [15]:

$$
\begin{aligned}
& \frac{\mathrm{d} r}{\mathrm{~d} t}=V \sin (\gamma) \\
& \frac{\mathrm{d} \theta}{\mathrm{d} t}=\frac{V \cos (\gamma) \sin (\psi)}{r \cos (\phi)} \\
& \frac{\mathrm{d} \phi}{\mathrm{d} t}=\frac{V \cos (\gamma) \cos (\psi)}{r} \\
& \frac{\mathrm{d} V}{\mathrm{~d} t}=-\frac{D}{m}-g \sin (\gamma)+\omega_{e} r^{2} \cos (\phi) \times[\sin (\gamma) \cos (\phi)-\cos (\gamma) \sin (\phi) \cos (\psi)] \\
& \frac{\mathrm{d} \gamma}{\mathrm{d} t}=\frac{1}{V}\left\{\frac{L \cos (\sigma)}{m}+\left(\frac{V^{2}}{r}-g\right) \cos (\gamma)+\omega_{e}^{2} r \cos (\phi)[\cos (\gamma) \cos (\phi)+\sin (\phi) \sin (\gamma) \cos (\psi)]\right\} \\
& \frac{\mathrm{d} \psi}{\mathrm{d} t}=\frac{1}{V}\left\{\frac{L \sin (\sigma)}{m \cos (\gamma)}+\frac{V^{2}}{r} \cos (\gamma) \sin (\psi) \tan (\phi)-2 \omega_{e} V[\tan (\gamma) \cos (\phi) \cos (\psi)-\sin (\phi)]\right. \\
& \left.+\frac{\omega_{e}^{2} r}{\cos (\gamma)} \sin (\phi) \cos (\phi) \sin (\psi)\right\}
\end{aligned}
$$


where $r$ is the radial distance from the center of the Earth to the RHV; $\theta$ and $\varphi$ are the longitude and the latitude, respectively; $V$ is the Earth-relative velocity; $\psi$ is the velocity heading angle relative to local north and $\gamma$ is the flight-path angle; $m$ and $\sigma$ are the mass of the vehicle and the BA, respectively; $D$ and $L$ are the aerodynamic drag force and lift force, respectively. They are related to the aerodynamic coefficients, AOA and velocity. They will be modeled in the following chapter. The angular rate of the Earth is denoted as $\omega_{e}$ and local gravitational acceleration is denoted as $g$, which yields $g=\mu / r^{2}$ with $\mu=3.986032 \times 10^{14} \mathrm{~m}^{3} / \mathrm{s}^{2}$ being the Earth's gravitational parameter.

The attitude dynamic model, with three degrees of freedom (DOFs), of the RHV in the re-entry phase is as follows [16]:

$$
\begin{aligned}
& \frac{\mathrm{d} \alpha}{\mathrm{d} t}=\omega_{y}-\tan (\beta)\left[\omega_{x} \cos (\alpha)+\omega_{z} \sin (\alpha)\right]+\frac{m g \cos (\gamma) \cos (\sigma)-L}{m V \cos (\beta)} \\
& \frac{\mathrm{d} \beta}{\mathrm{d} t}=\omega_{x} \sin (\alpha)-\omega_{z} \cos (\alpha)+\frac{g \cos (\gamma) \sin (\sigma)}{V} \\
& \frac{\mathrm{d} \sigma}{\mathrm{d} t}=\sec (\beta)\left[\omega_{x} \cos (\alpha)+\omega_{z} \sin (\alpha)\right] \\
& +\frac{1}{m V}\{-m g \cos (\gamma) \cos (\sigma) \tan (\beta)+L[\tan (\gamma) \sin (\sigma)+\tan (\beta)]\} \\
& \frac{\mathrm{d} \omega_{x}}{\mathrm{~d} t}=\frac{1}{I_{x x}}\left[M_{x}-\left(I_{z z}-I_{y y}\right) \omega_{y} \omega_{z}\right] \\
& \frac{\mathrm{d} \omega_{y}}{\mathrm{~d} t}=\frac{1}{I_{y y}}\left[M_{y}-\left(I_{x x}-I_{z z}\right) \omega_{x} \omega_{z}\right] \\
& \frac{\mathrm{d} \omega_{z}}{\mathrm{~d} t}=\frac{1}{I_{z z}}\left[M_{z}-\left(I_{y y}-I_{x x}\right) \omega_{x} \omega_{y}\right]
\end{aligned}
$$

where $\alpha, \beta$, and $\sigma$ are the AOA, sideslip angle, and BA, respectively; $\omega_{x}, \omega_{y}$, and $\omega_{z}$ are the roll, pitch, and yaw angular rates, respectively; Ixx, Iyy, Izz denote the moments of inertia. $M_{x}, M_{y}$, and $M_{z}$ denote the roll, pitch, and yaw moments acting on the RHV.

Equations (1) and (2) consist of the six-DOF dynamic models for RHV, which is the mathematic basis for the novel IGC scheme.

\subsection{Aerodynamic Characteristics}

The moments $M_{x}, M_{y}$, and $M_{z}$ and forces $L, D$ are provided by the actuators, typically control surfaces and the RCS. Here, only surfaces are considered. The moments and forces are related to air density, reference area, aerodynamic coefficients, etc., and they can be modeled as follows:

$$
\begin{aligned}
& {\left[\begin{array}{c}
M_{x} \\
M_{y} \\
M_{z}
\end{array}\right]=\left[\begin{array}{c}
q S_{r e f} b_{r e f} C_{m x} \delta_{x} \\
q S_{r e f} C_{r e f} C_{m y} \delta_{y} \\
q S_{r e f} b_{r e f} C_{m z} \delta_{z}
\end{array}\right]} \\
& {\left[\begin{array}{c}
L \\
D \\
Y
\end{array}\right]=\left[\begin{array}{c}
q S_{r e f} C_{L} \\
q S_{r e f} C_{D} \\
q S_{r e f} C_{Y}
\end{array}\right]}
\end{aligned}
$$

where $Y$ is the lateral force which is ignored in the motion equations and $q=0.5 \rho V^{2}$ is the dynamic pressure with $\rho=\rho_{0} e^{h / h s}$ being the atmospheric density. $\rho_{0}$ is the atmospheric density at sea level and $h s=7254.24 \mathrm{~m}$ is the scale height while $h$ is the current height of the RHV. $S_{r e f}$ is the reference area of the RHV. $b_{r e f}$ is the wingspan and $c_{r e f}$ is the mean geometric chord of the wing. $\delta_{x}, \delta_{y}$ and $\delta_{z}$ are equivalent deviations of surfaces, such as aileron, flap and tail. $C_{m x}, C_{m y}$ and $C_{m z}$ are moment coefficients while $C_{L}, C_{D}$ and $C_{Y}$ are force coefficients. These aerodynamic coefficients are generally related with AOA and velocity. To simplify the problem, only the force coefficients $C_{L}$ and $C_{D}$ ( $C_{Y}$ is ignored) are modeled and studied in the paper. Principles and methods are the same for moment coefficients. In the case of CAV-H developed by Lockheed Martin Space Systems Company [26], its $C_{L}$ and $C_{D}$ can be modeled as:

$$
\begin{aligned}
& C_{L}=C_{L 0}+C_{L 1} \alpha+C_{L 2} e^{C_{L 3} V} \\
& C_{D}=C_{D 0}+C_{D 1} \alpha^{2}+C_{D 2} e^{C_{D 3} V}
\end{aligned}
$$




\subsection{Multiple Constraints}

Multiple constraints must be considered for re-entry trajectory generation and guidance, including path constraints, terminal constraints and control (command) constraints.

(1) Path constraints

For flight safety, the re-entry trajectory should satisfy the path constraints of heating rate, load factor and dynamic pressure as follows [15]:

$$
\begin{aligned}
& \dot{Q}=K_{Q \sqrt{\rho}} V^{3.15} \leq \dot{Q}_{\max } \\
& n=\frac{\sqrt{L^{2}+D^{2}}}{m g} \leq n_{\max } \\
& q=0.5 \rho V^{2} \leq q_{\max }
\end{aligned}
$$

where $\dot{Q}_{\max }, n_{\max }$ and $q_{\max }$ are maximum heating rate, load factor and dynamic pressure, respectively. These are strong path constraints that must be satisfied and there is an optional soft path constraint for stable flight—namely, quasi-equilibrium glide constraint:

$$
\frac{\mu}{r^{2}}-\frac{V}{r}-\frac{L}{m} \leq 0
$$

(2) Terminal constraints

Terminal constraints vary greatly according to different missions. Typically, the terminal location must be within a certain threshold with a specific velocity and/or flight path angle. Terminal constraints for different trajectories will be specified in the simulation section.

(3) Control (command) constraints

In most research for re-entry trajectory generation and guidance, flow angles of AOA, $\mathrm{BA}$ and slide angle are set as controls or commands for the nonlinear optimization problems, and typically only the range constraints of flow angles are considered. However, since the flow angles are controlled by the actuators, which provide moments directly, the angular rate and acceleration of the flow angles must also be considered, otherwise the controls for the planned trajectory may not be available. In this paper, the controls of the GPM for trajectory generation are set as moments, while flow angles and angular rates of the RHV are extended in states. By using moments as controls, the first and second order of derivatives of flow angles can be constrained easily and at the same time we get rid of complex actuator configurations, making the method explicit and universal. By setting the ranges of the controls, the angular rate of attitudes and flow angles, more constraints have been imposed on flow angles, which further guarantee their accessibility. The constraints for moments, attitude angular rate and flow angles are as follows:

$$
\begin{aligned}
& \left|M_{x}\right| \leq M_{x \max },\left|M_{y}\right| \leq M_{y \max },\left|M_{z}\right| \leq M_{z \max } \\
& \left|\omega_{x}\right| \leq \omega_{x \max },\left|\omega_{y}\right| \leq \omega_{y \max },\left|\omega_{z}\right| \leq \omega_{z \max } \\
& \alpha_{\min } \leq \alpha \leq \alpha_{\max },|\beta| \leq \beta_{\max },|\sigma| \leq \sigma_{\max }
\end{aligned}
$$

\section{The Novel IGC Schemes}

The guidance and control systems of RHVs are usually designed separately and their mutual influences are ignored, so the synergistic relationships between the two subsystems are not fully exploited. In order to improve the guidance and control performance, a novel IGC scheme is proposed with the capabilities of online aerodynamic coefficient estimation based on a UKF and online trajectory generation based on the GPM. The whole diagram of IGC is illustrated in Figure 1. 


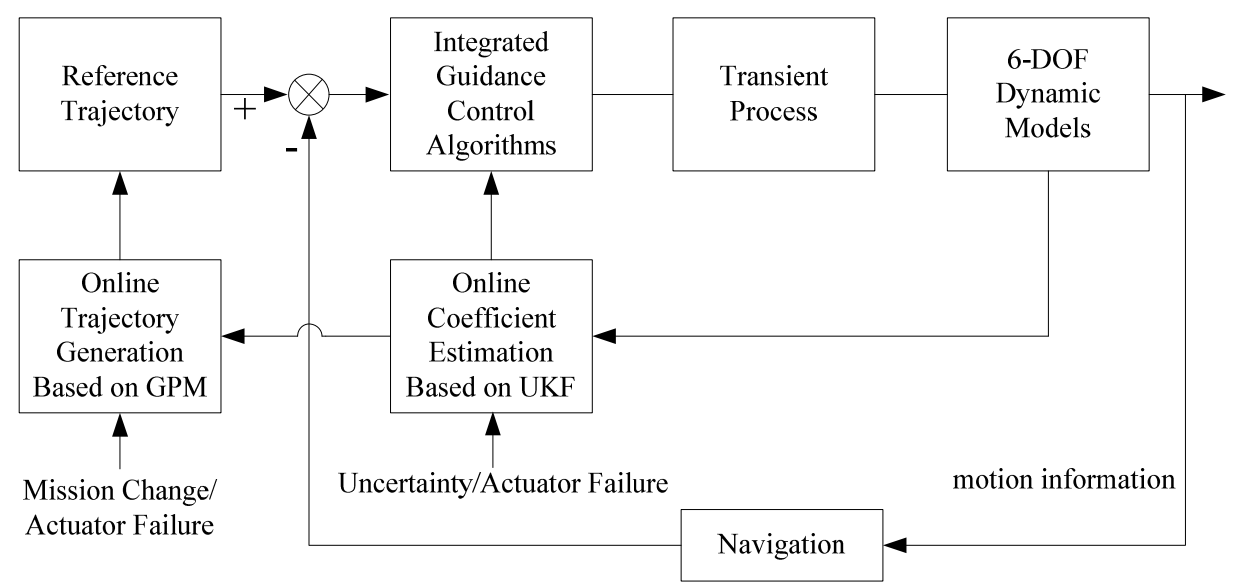

Figure 1. Diagram of the innovative integrated guidance and control (IGC) scheme proposed in the research.

\subsection{Online Trajectory Generation Based on the GPM}

Essentially, re-entry trajectory generation is a nonlinear optimization problem under various constraints. There are two main types of methods to solve the problem-a direct method and indirect method. The former turns continuous nonlinear problems into discrete nonlinear programing for solution. The GPM is one of the direct methods at a global scale that has been widely used in re-entry trajectory generation for its high accuracy and efficiency. The GPM discretizes the continuous states and commands, approximates them in the form of polynomials and satisfies the constraints at certain match points. As the procedure is conducted at a global scale, the parameters that need to be determined are less than traditional local approximation methods while the accuracy is higher.

In this paper, both point-mass and attitude dynamic models, namely Equations (1) and (2), are applied in trajectory generation based on the GPM. As stated previously, the controls (commands) are the moments of the actuators:

$$
u_{G P M}=\left[\begin{array}{lll}
M_{x} & M_{y} & M_{z}
\end{array}\right]^{\mathrm{T}}
$$

where the subscript $x, y$ and $z$ refer to the moment along the front side, right side and down side of the RHV body frame. The states are expanded to 12 dimensions:

$$
X_{G P M}=\left[\begin{array}{llllllllllll}
r & \theta & \varphi & V & \gamma & \psi & \alpha & \beta & \sigma & \omega_{x} & \omega_{y} & \omega_{z}
\end{array}\right]^{\mathrm{T}}
$$

Each state has its range limitation.

Two cost functions are designed in this paper: one is the maximum lateral range and the other is the least final point error at a specific position:

$$
\begin{aligned}
& J_{G P M 1}\left[X_{G P M}\left(t_{f}\right)\right]=-\varphi\left(t_{f}\right) \\
& J_{G P M 2}\left[X_{G P M}\left(t_{f}\right)\right]=\left[r\left(t_{f}\right)-r_{T}\right]^{2}+K_{\theta}\left[\theta\left(t_{f}\right)-\theta_{T}\right]^{2}+K_{\varphi}\left[\varphi\left(t_{f}\right)-\varphi_{T}\right]^{2}
\end{aligned}
$$

where $t_{f}$ is the time at terminal and $r_{T}, \theta_{T}$ and $\varphi_{T}$ represent the desired final location. $K_{\theta}$ and $K_{\varphi}$ are constant scale factors with units of meters.

The GPM will provide the numerical states and controls at each match point while their values between match points can be achieved by polynomial fitting with respect to time. The number of match points affects the computation load and accuracy at the same time. It is always true that the more match points the higher computation load, but this is not always true for accuracy. Therefore, the appropriate number of match points should be determined, especially for online trajectory generation which demands more for computation efficiency. Discussions will be scheduled in the simulation section. 
Moreover, unlike the offline scenario, the online trajectory is being generated when the RHV is guided according to the offline trajectory. The initial motion information used by the GPM is at time $t_{t g 0}$ and it takes $t_{\text {tgf }}$ to finish online trajectory generation, so the new trajectory is referenced by IGC from its time of $t_{t g f}$. This is also detailed in the simulations.

\subsection{Online Aerodynamic Coefficient Estimation Based on UKF}

As mentioned previously, the maximum and average errors of the fitting model for aerodynamic coefficients $C_{L}$ and $C_{D}$ are $12 \%$ and $3.8 \%$, respectively. Additionally, in the case of actuator failure, the aerodynamic coefficients may change greatly. To deal with the failure problem and improve the IGC performance, the aerodynamic coefficients should be estimated online.

Because both the coefficient model and motion model are strongly nonlinear, a UKF is adopted in our scheme due to its better accuracy and lower complexity for online application, since the Jacobian matrices are not needed.

(1) State functions

The state of a UKF has 12 elements:

$$
X_{U K F}=\left[\begin{array}{llllllllllll}
r & \theta & \varphi & V & \gamma & \psi & \eta_{D 0} & \eta_{D 1} & \eta_{D 2} & \eta_{L 0} & \eta_{L 1} & \eta_{L 2}
\end{array}\right]^{\mathrm{T}}
$$

The first six elements are the same with $X_{G P M}$ while the last six elements are the aerodynamic coefficient factors ranging from 0 to 1 , which indicate the extent that the aerodynamic coefficients have changed. According to Equation (4):

$$
\begin{aligned}
& C_{L}=\eta_{L 0} C_{L 0}+\eta_{L 1} C_{L 1} \alpha+\eta_{L 2} C_{L 2} e^{C_{L 3} V} \\
& C_{D}=\eta_{D 0} C_{D 0}+\eta_{D 1} C_{D 1} \alpha^{2}+\eta_{D 2} C_{D 2} e^{C_{D 3} V}
\end{aligned}
$$

In fact, the coefficients $C_{L 3}$ and $D_{L 3}$ have much lower impacts on $C_{L}$ and $C_{D}$, which is detailed in Section 4, so their changing factors are not included in the states.

Assuming the factors are random constants or slowly changing values, they are modeled as follows:

$$
\dot{\eta}_{D i}=0, \quad \dot{\eta}_{L i}=0(i=0,1,2)
$$

The input vector consists of AOA $\alpha$ and $\mathrm{BA} \sigma$ with the assumption that the sideslip angle is zero:

$$
U_{U K F}=\left[\begin{array}{cc}
\alpha & \sigma
\end{array}\right]^{\mathrm{T}}
$$

$\alpha$ affects the $C_{L}$ and $C_{D}$ together with velocity and aerodynamic coefficients. Therefore, they determine the lift and drag forces $L$ and $D . \sigma$ directly affects the velocity heading angle $\psi$ and flight path angle $\gamma$ according to Equation (1). So, $\alpha$ and $\sigma$ are chosen as inputs.

The three-DOF point-mass dynamic function and the coefficient factor function together constitute the nonlinear system state function. After discretization, the state transition function $F_{U K F}$ yields:

$$
X_{U K F}^{k}=F_{U K F}\left(X_{U K F}^{k-1}, U_{U K F}^{k-1}\right)+W_{U K F}^{k-1}
$$

where $k$ is the discretized time while $W_{U K F}$ is the zero-mean unrelated Gaussian process noise vector with a variation matrix of $Q_{U K F}$.

(2) Measurement functions

The measurements are the radial distance from the center of the Earth to the RHV, $r$; the longitude and the latitude, $\theta$ and $\varphi$; the Earth-relative velocity $V$ :

$$
Z_{U K F}=\left[\begin{array}{llll}
r & \theta & \varphi & V
\end{array}\right]^{\mathrm{T}}
$$


These measurements can be obtained through navigation systems such as an inertial navigation system, Global Satellite Navigation System, etc. The measurement function is linear so the measurement matrix can be shown as:

$$
H_{U K F}=[\operatorname{eye}(4) \quad \operatorname{zeros}(4,8)]
$$

where eye(4) is four-dimensional unit matrix. The measurement function can then be expressed as:

$$
Z_{U K F}=H_{U K F} X_{U K F}+V_{U K F}
$$

where $V_{U K F}$ is the zero-mean unrelated Gaussian measurement noise vector with a variation matrix of $R_{U K F}$.

The coefficient estimation can then be realized through the UKF algorithm. The estimated coefficients will be used for online trajectory generation and IGC. Parameters of UKF will be introduced in Section 4.

\subsection{IGC Laws Based on $L Q R$ and SMC}

Traditionally, the guidance system and attitude control system are designed separately. The guidance outputs command according to the current motion information, generally in the form of flow angles such as AOA and BA. The attitude control system just follows the commands as best as possible and no feedback is provided to the guidance system. However, as guidance and control systems are highly interactive, their mutual influences should be considered and the design should be integrated.

In our research, the principle of spectrum separation is applied for the IGC design so that the attitude control system is the inner loop with a small time constant while the guidance system is the outer loop with a large time constant. The trajectory tracking guidance laws based on the LQR and attitude control method based on two-layer SMC are designed. The guidance laws generate commands under the control capability constraints and attitude tracking errors will be fed back to the guidance system for command updates.

(1) Guidance laws based on LQR

LQRs can be applied to multi-input and multioutput control systems with the advantages of high accuracy, high efficiency and easy realization online. For re-entry trajectory tracking guidance, the dynamic models need to be linearized for the LQR. Generally, only the three-DOF dynamic model is applied and AOA and BA are the commands or inputs. In our IGC scheme, with the same thought of trajectory generation based on the GPM, we take the ability of the attitude control system into consideration by applying the six-DOF dynamic model and setting moments as commands. Therefore, the LQR based guidance system can also provide expected flow angles and their rates for an attitude control system based on an SMC.

The state of LQR has 12 elements that are the differences between the reference trajectory and the actual one.

$$
\begin{aligned}
& \Delta X_{L Q R}=\left[\begin{array}{cccccc}
\Delta r & \Delta \theta & \Delta \varphi & \Delta V & \Delta \gamma & \Delta \psi \\
\Delta \alpha & \Delta \beta & \Delta \sigma & \Delta \omega_{x} & \Delta \omega_{y} & \Delta \omega_{z}
\end{array}\right]
\end{aligned}
$$

The commands of the LQR are the moment differences that need to be updated:

$$
\Delta U_{L Q R}=\left[\begin{array}{ccc}
\Delta M_{x} & \Delta M_{y} & \Delta M_{z}
\end{array}\right]^{\mathrm{T}}
$$

At any point of the trajectory, the linearized system function can be written as:

$$
\Delta \dot{X}_{L Q R}=A_{L Q R} \Delta X_{L Q R}+B_{L Q R} \Delta U_{L Q R}
$$


where $A_{L Q R}$ and $B_{L Q R}$ are the state matrix and command matrix, yielding:

$$
\begin{gathered}
A_{L Q R}=\left[\begin{array}{ccc}
\frac{\partial F_{1}}{\partial \Delta X_{L Q R}(1)} & \cdots & \frac{\partial F_{1}}{\partial \Delta X_{L Q R}(12)} \\
\cdots & \cdots & \cdots \\
\frac{\partial F_{12}}{\partial \Delta X_{L Q R}(1)} & \cdots & \frac{\partial F_{12}}{\partial \Delta X_{L Q R}(12)}
\end{array}\right] \\
B_{L Q R}=\left[\begin{array}{ccc}
\frac{\partial F_{1}}{\partial \Delta U_{L Q R}(1)} & \cdots & \frac{\partial F_{1}}{\partial \Delta U_{L Q R}(3)} \\
\cdots & \cdots & \cdots \\
\frac{\partial F_{12}}{\partial \Delta U_{L Q R}(1)} & \cdots & \frac{\partial F_{12}}{\partial \Delta U_{L Q R}(3)}
\end{array}\right]
\end{gathered}
$$

$F_{i}(i=1,2 \ldots 12)$ are the expressions at the right side of the equal sign in Equations (1) and (2).

The LQR calculated $\Delta U_{L Q R}$ to tune $\Delta X_{L Q R}$ so that the minimum of the cost function can be reached (subscript $L Q R$ of $\Delta X_{L Q R}$ and $\Delta U_{L Q R}$ is omitted):

$$
J_{L Q R}=\lim _{t \rightarrow \infty} \int_{t_{0}}^{t_{f}}\left(\Delta X^{\mathrm{T}} Q_{L Q R} \Delta X+\Delta U^{\mathrm{T}} R_{L Q R} \Delta U\right) \mathrm{d} t
$$

where $Q_{L Q R}$ and $R_{L Q R}$ are non-negative scale matrices that can be determined by Bryson's rule.

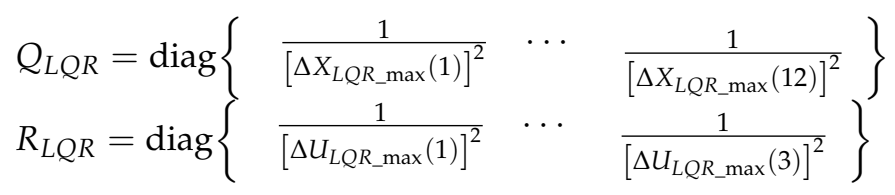

The subscript max means the maximum of each element. In other words, each element is expected within these setting ranges.

The updated command can be calculated as:

$$
\Delta U_{L Q R}=\left(R_{L Q R}\right)^{-1}\left(B_{L Q R}\right)^{\mathrm{T}} P_{L Q R} \cdot \Delta X_{L Q R}
$$

where $P_{L Q R}$ is derived from the Riccati equation as follows (all $L Q R$ subscripts are omitted):

$$
(A)^{\mathrm{T}} P+P A-P B(R)^{-1}(B)^{\mathrm{T}} P+Q=0
$$

Thus, guidance LQR updates the moments to control RHV and tracks the reference trajectory. Moreover, as the LQR state contains the expected flow angle and angular rate information, they can be provided for the attitude control system.

(2) Attitude control method based on SMC

A second-layer SMC is designed for RHV attitude control in the novel IGC scheme. Based on the expected angular rates in body frame and flow angles, ignoring the velocityrelated terms in the first three equations in Equation (2), the rate of flow angles can be derived:

$$
\begin{aligned}
& \dot{\alpha}=\omega_{y}-\omega_{x} \cos (\alpha) \tan (\beta)-\omega_{z} \sin (\alpha) \tan (\beta) \\
& \dot{\beta}=\omega_{x} \sin (\alpha)-\omega_{z} \cos (\alpha) \\
& \dot{\sigma}=\left[\omega_{x} \cos (\alpha)+\omega_{z} \sin (\alpha)\right] \sec (\beta)
\end{aligned}
$$

The guidance outer loop provides the expected flow angles and their angular rates and they become the commands for the attitude control inner loop.

The first-layer slide mode surface of the attitude SMC is designed based on the flow angle commands:

$$
S_{0}=\left[\begin{array}{l}
S_{01} \\
S_{02} \\
S_{03}
\end{array}\right]=\left[\begin{array}{l}
e_{\alpha} \\
e_{\beta} \\
e_{\sigma}
\end{array}\right]=\left[\begin{array}{l}
\alpha-\alpha_{c} \\
\beta-\beta_{c} \\
\sigma-\sigma_{c}
\end{array}\right]
$$


Exponent approaching is adopted as follows:

$$
\dot{S}_{0}=-K_{01} S_{0}-K_{02}\left[\begin{array}{c}
\operatorname{sign}\left(S_{01}\right) \\
\operatorname{sign}\left(S_{02}\right) \\
\operatorname{sign}\left(S_{03}\right)
\end{array}\right]
$$

where $K_{01}$ and $K_{02}$ are positive feedback factors and sign() is sign function. Using the set virtual angular rate in the body frame as commands and according to Equations (27)-(29), it can be shown that:

$$
\left[\begin{array}{c}
\omega_{x c} \\
\omega_{y c} \\
\omega_{z c}
\end{array}\right]=\left[\begin{array}{ccc}
-\cos (\alpha) \tan (\beta) & 1 & -\sin (\alpha) \tan (\beta) \\
\sin (\alpha) & 0 & -\cos (\alpha) \\
\cos (\alpha) \sec (\beta) & 0 & \sin (\alpha) \sec (\beta)
\end{array}\right]^{-1}\left\{-K_{01} S_{0}-K_{02}\left[\begin{array}{c}
\operatorname{sign}\left(S_{01}\right) \\
\operatorname{sign}\left(S_{02}\right) \\
\operatorname{sign}\left(S_{03}\right)
\end{array}\right]+\left[\begin{array}{c}
\dot{\alpha}_{c} \\
\dot{\beta}_{c} \\
\dot{\sigma}_{c}
\end{array}\right]\right\}
$$

The command of angular rates of flow angles $\left[\begin{array}{lll}\dot{\alpha}_{c} & \dot{\beta}_{c} & \dot{\sigma}_{c}\end{array}\right]$ can be derived from guidance system according to Equation (27).

Based on virtual angular rates, the second-layer sliding surface is designed as follows:

$$
S_{1}=\left[\begin{array}{l}
S_{11} \\
S_{12} \\
S_{13}
\end{array}\right]=\left[\begin{array}{c}
\omega_{x}-\omega_{x c} \\
\omega_{y}-\omega_{y c} \\
\omega_{z}-\omega_{z c}
\end{array}\right]
$$

Similarly, exponent approaching is adopted:

$$
\dot{S}_{1}=-K_{11} S_{1}-K_{12}\left[\begin{array}{c}
\operatorname{sign}\left(S_{11}\right) \\
\operatorname{sign}\left(S_{12}\right) \\
\operatorname{sign}\left(S_{13}\right)
\end{array}\right]
$$

where $K_{01}$ and $K_{02}$ are positive feedback factors. Therefore, the moment commands for attitude control can be derived as:

$$
\left[\begin{array}{l}
M_{x c} \\
M_{y c} \\
M_{z c}
\end{array}\right]=\left[\begin{array}{l}
\left(I_{z z}-I_{y y}\right) \omega_{y} \omega_{z} \\
\left(I_{x x}-I_{z z}\right) \omega_{x} \omega_{z} \\
\left(I_{y y}-I_{x x}\right) \omega_{x} \omega_{y}
\end{array}\right]+\left[\begin{array}{ccc}
I_{x x} & 0 & 0 \\
0 & I_{y y} & 0 \\
0 & 0 & I_{z z}
\end{array}\right] \times\left\{-K_{11} S_{1}-K_{12}\left[\begin{array}{c}
\operatorname{sign}\left(S_{11}\right) \\
\operatorname{sign}\left(S_{12}\right) \\
\operatorname{sign}\left(S_{13}\right)
\end{array}\right]+\left[\begin{array}{c}
\dot{\omega}_{x c} \\
\dot{\omega}_{y c} \\
\dot{\omega}_{z c}
\end{array}\right]\right\}
$$

The virtual command of the second layer $\left[\begin{array}{ccc}\dot{\omega}_{x c} & \dot{\omega}_{y c} & \dot{\omega}_{z c}\end{array}\right]$ does not affect the control performance as much and they can be set to zero.

Finally, a transient process is considered where the actuators are modeled as secondorder inertial element with the response frequency of $10 \pi$ and damping ratio of 0.707 .

\section{Simulations and Verifications}

Comprehensive simulations were conducted to verify the effectiveness and advantages of the proposed IGC scheme. Firstly, a reference trajectory was generated offline with the GPM and its cost function was $J_{G P M 1}$ - namely, the largest lateral range. If the dynamic coefficients are accurate, the IGC guarantees that RHV follow the trajectory with small errors. However, if the coefficients have errors or the actuator failure happens, it can be seen the performance of IGC obviously degrades for the offline reference trajectory, even if coefficient estimation online is applied. Finally, based on the online coefficient estimation, a new trajectory was generated to a specific final position. The IGC performance is comparable with that of accurate coefficients for offline trajectory.

\subsection{Configurations of the Simulations}

Some constants and aerodynamic coefficients in the simulations are listed as follows. The parameters of CAV-H, developed by Lockheed Martin Space Systems Company of USA, are adopted in the simulations and related parameters are listed in Table 1 [26]. 
Table 1. Constants and aerodynamic coefficients.

\begin{tabular}{|c|c|c|}
\hline Symbol & Term & Value \\
\hline$R_{e}$ & Radius of the Earth & $6,378,000 \mathrm{~m}$ \\
\hline$\rho_{0}$ & $\begin{array}{c}\text { Atmospheric density at sea } \\
\text { level }\end{array}$ & $1.2258 \mathrm{~kg} / \mathrm{m}^{3}$ \\
\hline$\mu$ & Earth gravitational constant & $3.98603 \times 10^{14} \mathrm{~m}^{3} / \mathrm{s}^{2}$ \\
\hline$\omega_{e}$ & Earth rotation speed & $7.2922 \times 10^{-5} \mathrm{rad} / \mathrm{s}$ \\
\hline$m$ & mass of the RHV & $907.2 \mathrm{~kg}$ \\
\hline$S$ & Reference area & $0.4839 \mathrm{~m}^{2}$ \\
\hline$I_{x x}$ & Moment of inertia X & $125.2 \mathrm{~kg} \mathrm{~m}^{2}$ \\
\hline$I_{y y}$ & Moment of inertia $Y$ & $278.5 \mathrm{~kg} \mathrm{~m}^{2}$ \\
\hline$I_{z z}$ & Moment of inertia Z & $193.8 \mathrm{~kg} \mathrm{~m}^{2}$ \\
\hline$n_{\max }$ & Maximum load & 15 \\
\hline$q_{\max }$ & Maximum dynamic pressure & $5 \times 10^{4} \mathrm{~N} / \mathrm{m}^{2}$ \\
\hline$\dot{Q}_{\max }$ & Maximum heating rate & $8 \times 10^{5} \mathrm{~kW} / \mathrm{s}$ \\
\hline$K_{Q}$ & Heating parameter & $7.9686 \times 10^{-5}$ \\
\hline$C_{D 0}$ & \multirow{4}{*}{ Drag coefficients } & 0.0234 \\
\hline$C_{D 1}$ & & 2.3795 \\
\hline$C_{D 2}$ & & 0.3983 \\
\hline$C_{D 3}$ & & $-1.0794 \times 10^{-3}$ \\
\hline$C_{L 0}$ & \multirow{4}{*}{ Lift coefficients } & -0.2355 \\
\hline$C_{L 1}$ & & 2.9451 \\
\hline$C_{L 2}$ & & 0.2949 \\
\hline$C_{L 3}$ & & $-3.3943 \times 10^{-4}$ \\
\hline
\end{tabular}

\subsection{Simulation in Normal Conditions}

A trajectory of the largest lateral range with a cost function of $J_{G P M 1}$, as Equation (10) shows, is generated offline, as Figure 2 shows. Figure 2 a shows the three-dimension position. Figure $2 \mathrm{~b}$ shows the profiles of longitude, latitude, height and speed with respect to time. Figure $2 c$ shows the profiles of flight-path angle, velocity heading angle, AOA and BA with respect to time.

The initial height of RHV is $60 \mathrm{~km}$ and position is $(0,0)$. The initial velocity is $6400 \mathrm{~m} / \mathrm{s}$ and its heading is 90 degree-namely, flying towards the East. The maximum lateral range reaches about $45.4^{\circ} \mathrm{N}$ in $2110 \mathrm{~s}$. Terminal constraints are that the final height is $24 \mathrm{~km}$ and final velocity is $760 \mathrm{~m} / \mathrm{s}$ with the path angle of -3 degree, which are requirements from the horizontal landing phase of RHV.

As for the parameters of IGC system, the diagonal elements of $Q_{L Q R}$ are $(1 / 1000)^{2}$, $(\operatorname{Re} / 1000)^{2},(\operatorname{Re} / 1000)^{2},(1 / 30)^{2},(1800 / \pi)^{2},(1800 / \pi)^{2},(18 / \pi)^{2},(18 / \pi)^{2},(18 / \pi)^{2},(18 / 5 \pi)^{2}$, $(18 / 5 \pi)^{2}$, and $(18 / 5 \pi)^{2}$, respectively, while the diagonal elements of $R_{L Q R}$ are $(45 / \pi)^{2}$, $(180 / \pi)^{2}$, and $(90 / \pi)^{2}$, respectively. The GMC parameters $K_{01}, K_{02}, K_{11}$, and $K_{12}$ are 0.012 , $0.0015,13$, and 0.001 , respectively.

In normal conditions, the dynamic coefficients are all accurate and disturbances are small, the IGC based on LQR and GMC is capable of leading the vehicle to the target location with height and position error within $120 \mathrm{~m}$ while velocity error within $0.4 \mathrm{~m} / \mathrm{s}$, as Figure 3 shows. The simulation verifies the effectiveness of IGC algorithms based on LQR and GMC under normal conditions. 


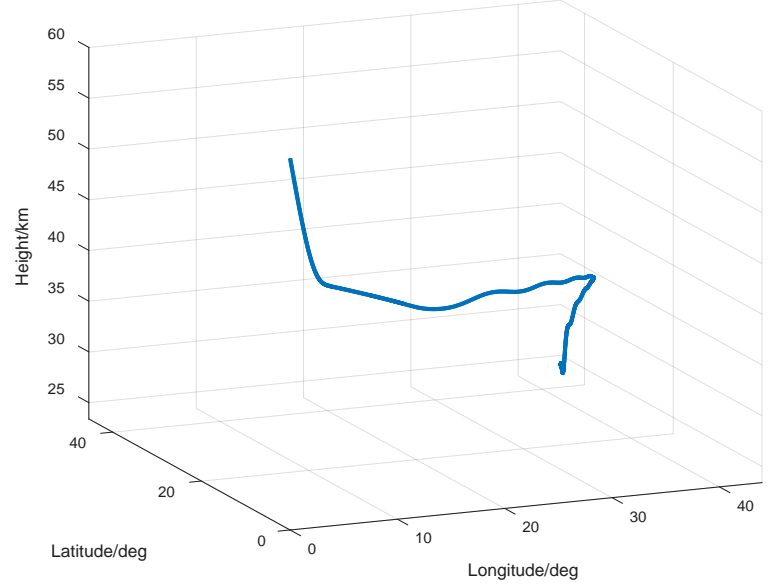

(a)
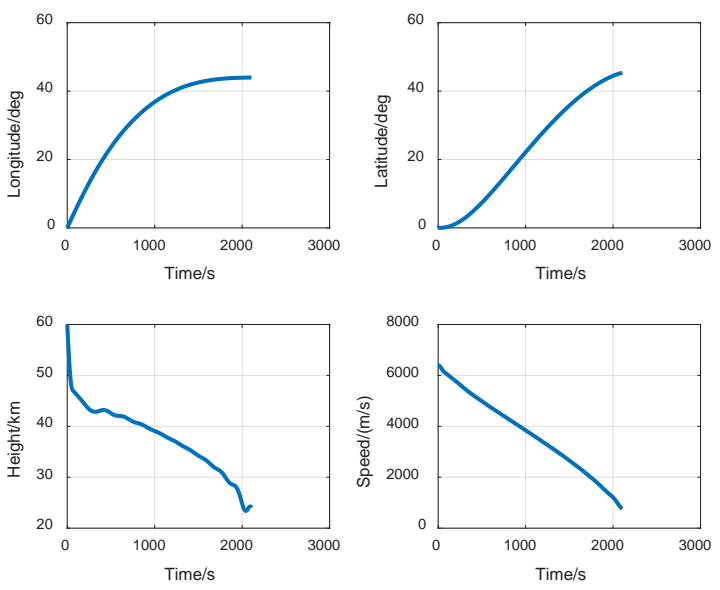

(b)
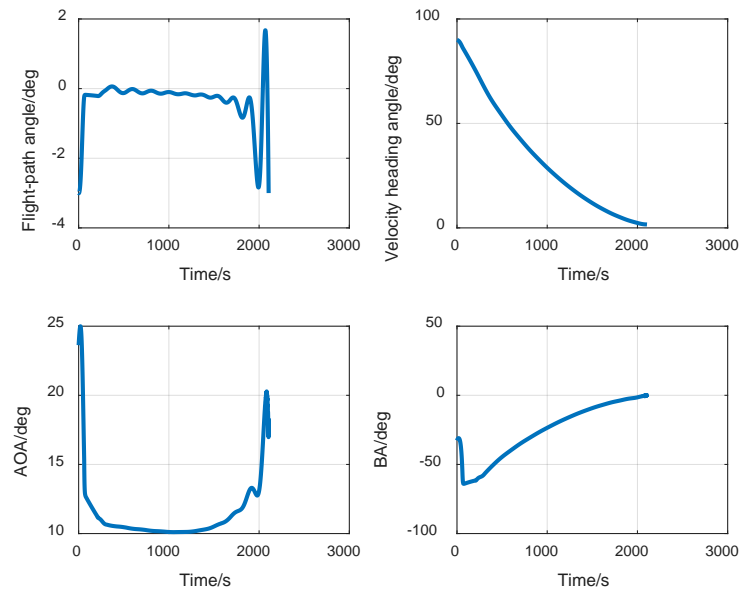

(c)

Figure 2. (a) Offline trajectory 3-dimension position, (b) offline trajectory profiles of longitude, latitude, height and speed with respect to time, (c) offline trajectory profiles of flight-path angle, velocity heading angle, angle of attack (AOA) and bank angle (BA) with respect to time. 

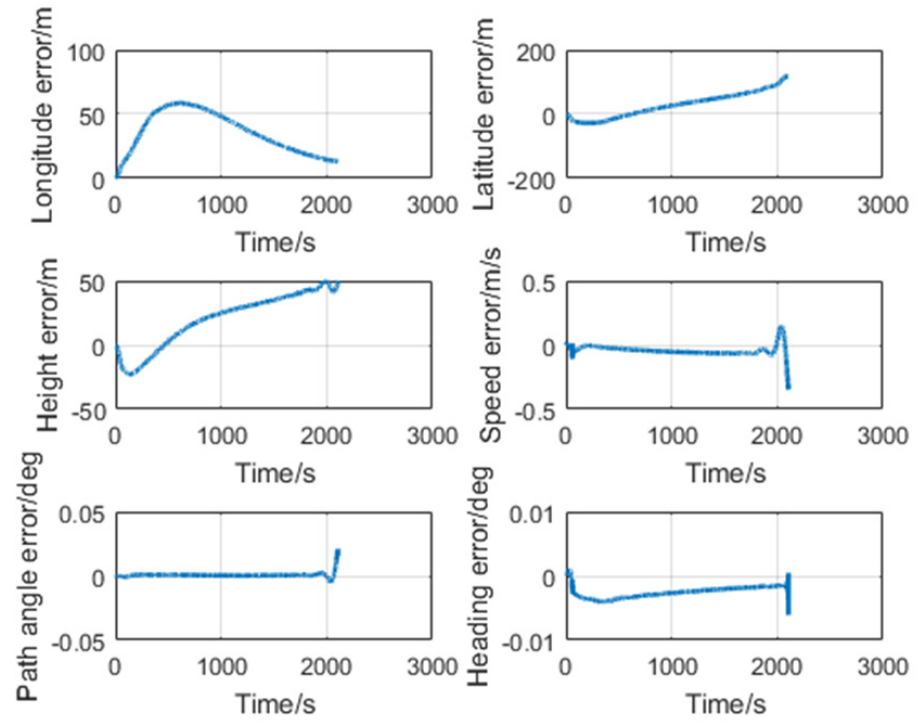

Figure 3. Offline trajectory position, velocity, flight path and heading angle errors under normal conditions.

\subsection{Simulation with Large Dynamic Coefficients Errors}

However, when dynamic coefficients have large errors or actuator failure happens, the performance of same IGC system degrades obviously. In this simulation, $10 \%$ of $C_{D 0}$ error and $-20 \%$ of $C_{L 0}$ error are added and the IGC results are shown in Figure 4.
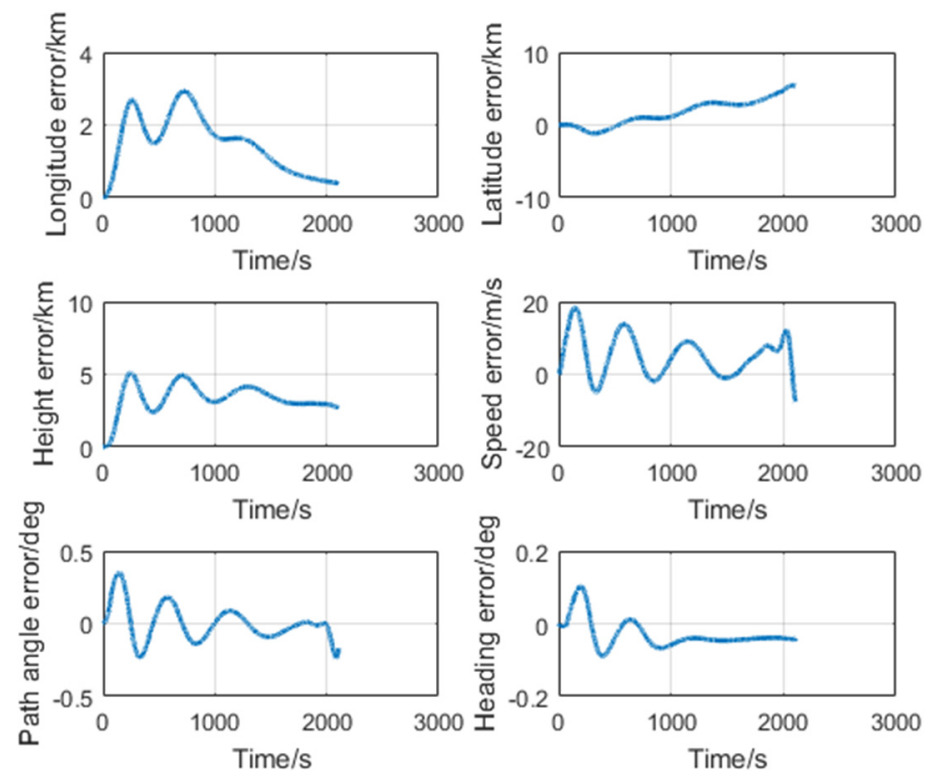

Figure 4. Offline trajectory position, velocity, flight path and heading angle errors with large coefficient errors.

According to the offline reference trajectory, the final height error is $2700 \mathrm{~m}$, with a position error of up to $5500 \mathrm{~m}$ and velocity error of $5 \mathrm{~m} / \mathrm{s}$, which are much larger than those in normal conditions. There are two reasons for this:

(1) The offline reference trajectory is generated based on accurate coefficients and it may not be reachable for the degraded flight ability;

(2) The guidance system also uses aerodynamic coefficients to update commands, which are also erroneous if coefficients are not accurate. 
The results of the dynamic coefficient estimation based on UKF are provided in Figure 5.

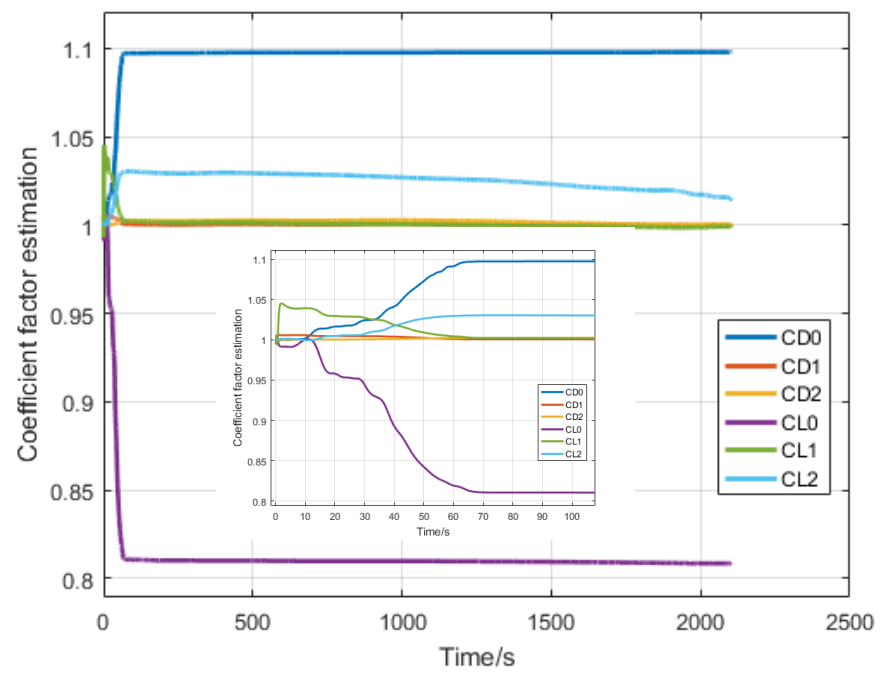

Figure 5. Result of dynamic coefficient estimation based on Unscented Kalman Filter (UKF).

The estimation begins from the very start of the flight and the accuracy of all coefficients is within $1 \%$ after about $70 \mathrm{~s}$ except from the $C_{L 2}$ factor $\eta_{L 2}$ because it has lower impact on the lift coefficient and its observability is lower. For example, when the AOA is 10 degrees and the velocity is $6000 \mathrm{~m} / \mathrm{s}$, the $C_{L}=0.3170$ while the part of $C_{L 2} e^{C_{L 3} V}$ is only 0.038 - namely, $12 \%$ of the whole efficient. Thus, the error of $2.5 \%$ of $C_{L 2}$ in this simulation leads to only $0.3 \%$ error of the whole efficient. In total, the estimation accuracies of $C_{L}$ and $C_{D}$ after $100 \mathrm{~s}$ are $0.86 \%$ and $0.75 \%$, respectively. Although the estimation based on UKF can provide accurate dynamic coefficients, the final position and velocity errors are still large due to reason (1), detailed previously. Therefore, once the coefficient change detected or mission profile changed, the online trajectory is required.

\subsection{Simulation with Online Trajectory Generation}

The number of GPM match points affects the computation load and accuracy at the same time. For the online scenario, more match points can be tolerated as time limitation is weak. For the online scenario, the new trajectory must be generated within seconds otherwise RHV may be in a dangerous situation with the potential of actuator failure. In our research, maximum match points for offline is 200 while for online the number is limited to 100. Indeed, in the simulations, the number of match points for offline is 186 and for online is 89 . The GPM is complemented by Matlab script on our computer with Intel Core i5-2520M CPU @2.5 GHz and a Windows 7 operating system and it takes $5.8 \mathrm{~s}$ to generate the online trajectory with cost function of $J_{G M P 2}$, which guides the RHV to the specific location of $(50 \mathrm{E}, 25 \mathrm{~N}, 30 \mathrm{~km})$ for emergency.

As the coefficient estimation settles down at about $100 \mathrm{~s}$, the online trajectory generation is scheduled to begin at the 120th s of the whole flight. Ten seconds are left for online trajectory generation. From the very beginning of the flight to the 130th s, the RHV was guided according to the offline trajectory. The initial motion information at the 120th s was used for online trajectory generation, but the reference trajectory was switched to the online one at the 130th s. In practice, the ten seconds are left for online GPM based trajectory computation.

Figure 6a shows the comparisons of the 3D positions of the online trajectory and the offline one. The IGC led the RHV according to the online trajectory for a new target after the 130th s. Figure $6 \mathrm{c}$ shows the 3D positions, velocity, path angle, heading, AOA and BA profiles. AOA ranges from 10 to 30 degrees while its limitations are -5 to 35 degrees. BA ranges from -72 to 35 degrees while its limitations are -89 to 89 degrees. The angular 
velocity of $\mathrm{AOA}$ and $\mathrm{BA}$ are mostly within $1 \mathrm{deg} / \mathrm{s}$ apart from the time of switched reference trajectory. Figure $6 \mathrm{~d}$ shows the angular velocities and accelerations of AOA and BA. Since moment constraints are considered, it can be seen that the angular acceleration of $\mathrm{AOA}$ and $\mathrm{BA}$ are within 0.4 and $2 \mathrm{deg} / \mathrm{s}^{2}$, respectively, which ensures that the flow angle commands are smooth and achievable.

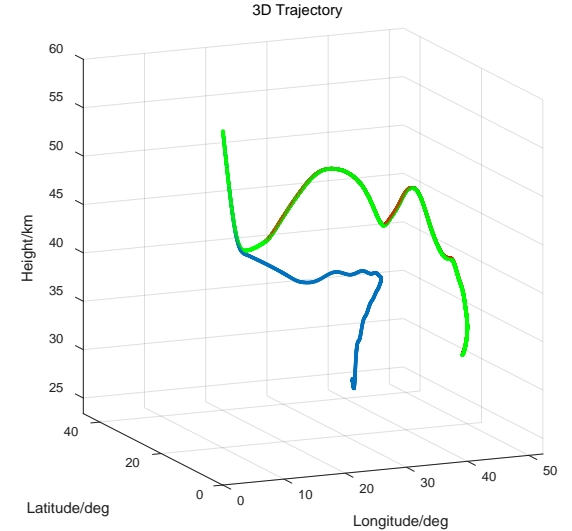

(a)
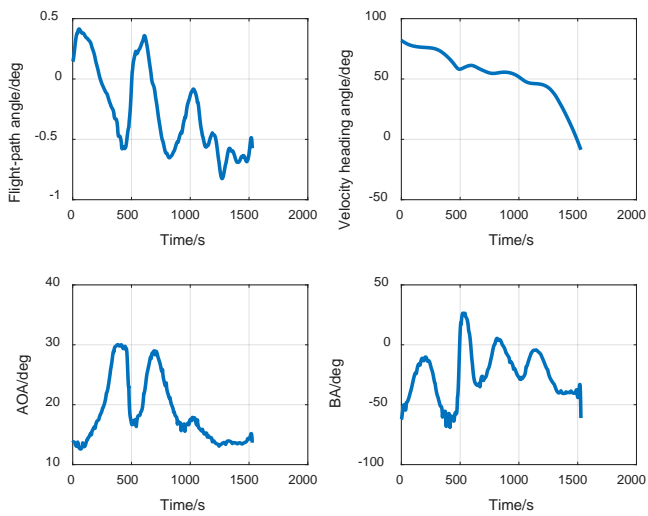

(c)
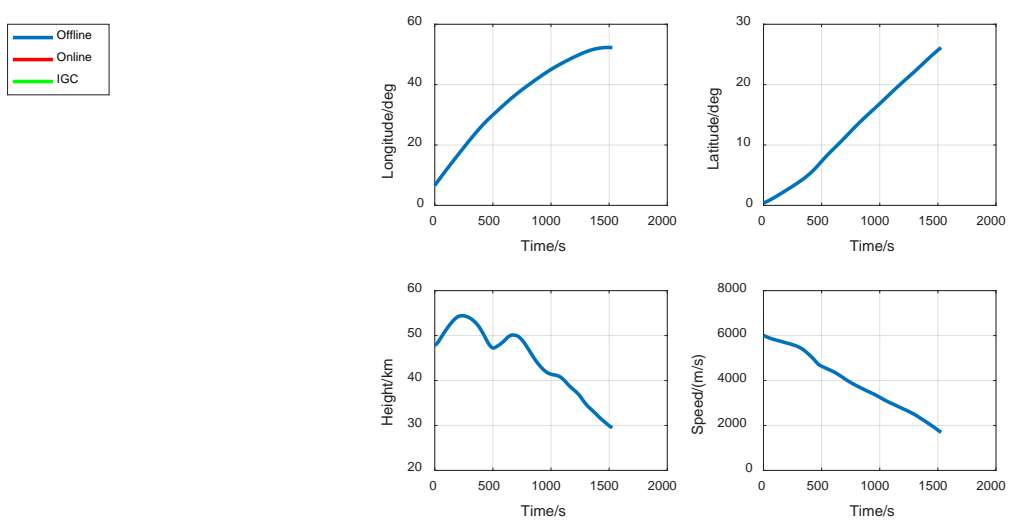

(b)
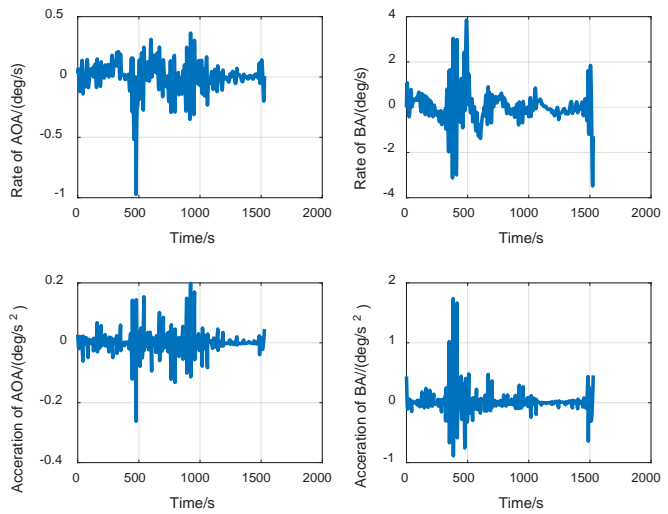

(d)

Figure 6. (a) Comparisons of the 3D positions of the online trajectory and the offline one. (b) Online trajectory profiles of longitude, latitude, height and speed with respect to time. (c) Online trajectory profiles of flight-path angle, velocity heading angle, AOA and BA with respect to time. (d) Online trajectory profiles of angular rates and accelerations of AOA and BA with respect to time.

Figure 7 shows that the online trajectory satisfied all the path constraints during the whole flight. The RHV passed through the velocity-height corridor perfectly.

The IGC errors at the final point under large coefficient errors according to online trajectory are similar with those in normal conditions according to offline trajectory-namely, the results described in Section 4.2. The height and position errors are within $200 \mathrm{~m}$ while velocity error is within $0.5 \mathrm{~m} / \mathrm{s}$, which is depicted in Figure 8 . 


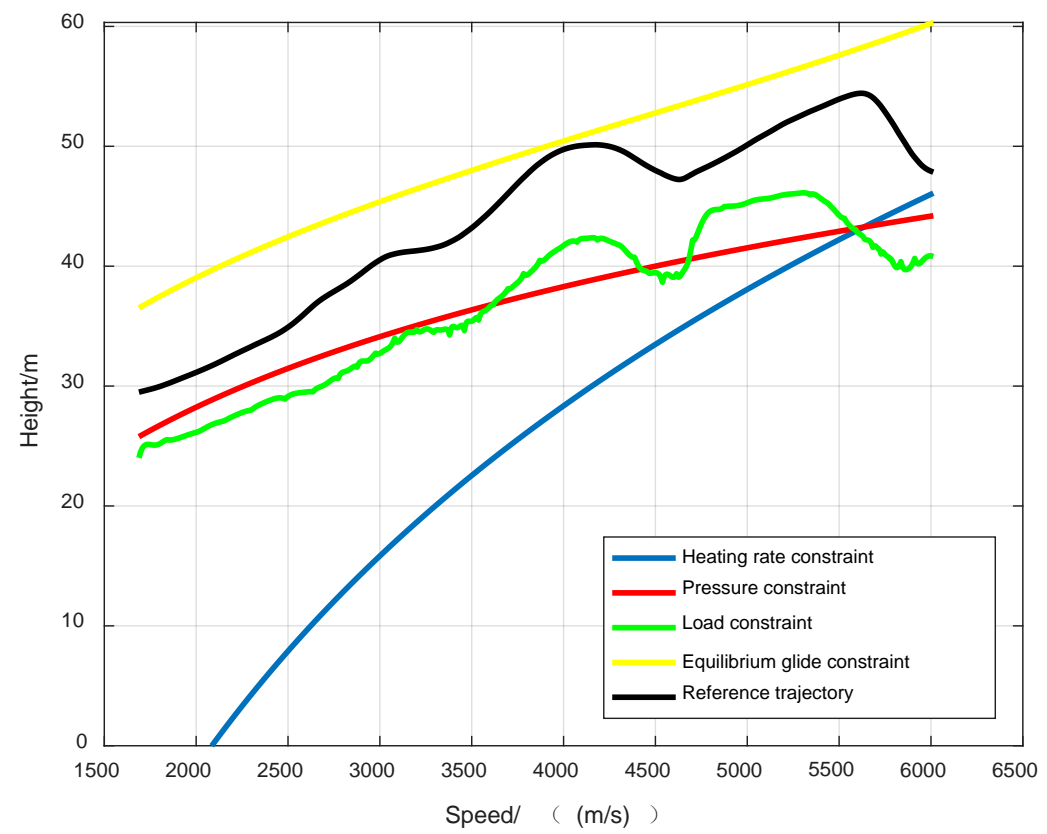

Figure 7. Online trajectory path constraints and velocity-height corridor.
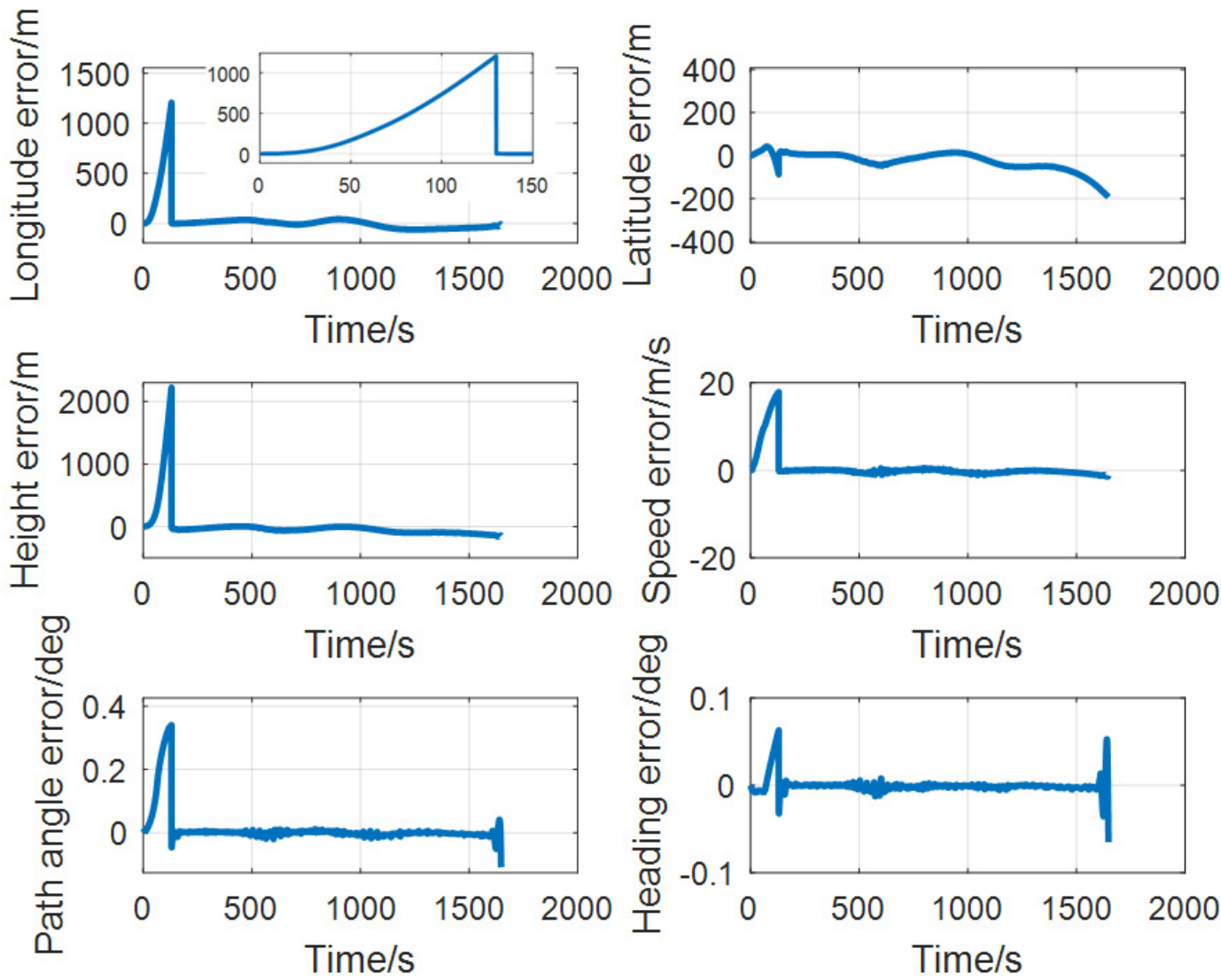

Figure 8. Online trajectory position, velocity, flight path and heading angle errors with large coefficient errors.

Figure 9a shows that the AOA and BA provided by guidance system are basically the same with reference trajectory. The difference comes from the LQR based guidance system for the purpose of eliminating motion error, especially when the online trajectory is initially referenced. The enlarged Figure $9 \mathrm{~b}$ shows that the attitude control system keeps the $\mathrm{AOA}$ and $\mathrm{BA}$ in accordance with guidance system states. The standard deviation of 
attitude error is within $0.05 \mathrm{deg}$, which verifies the effectiveness of attitude control system based on GMC.
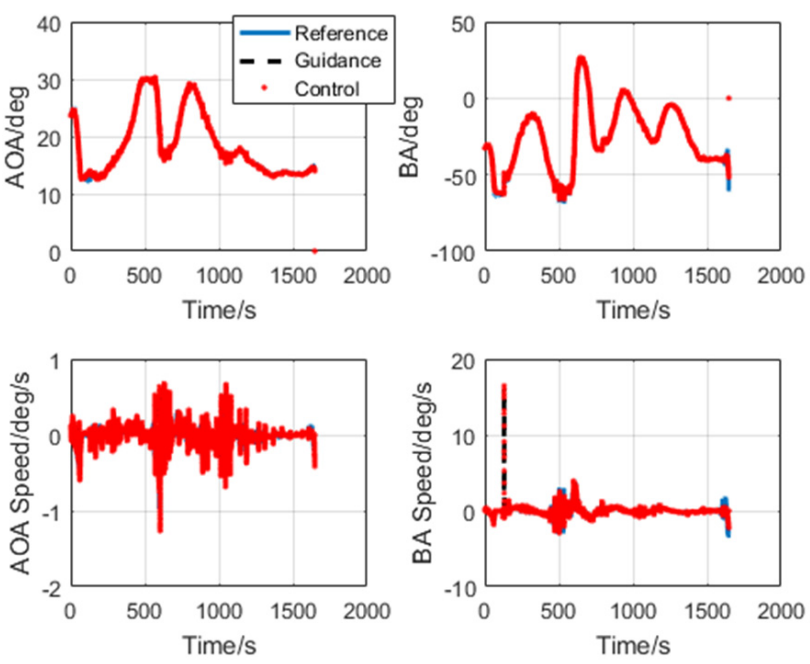

(a)
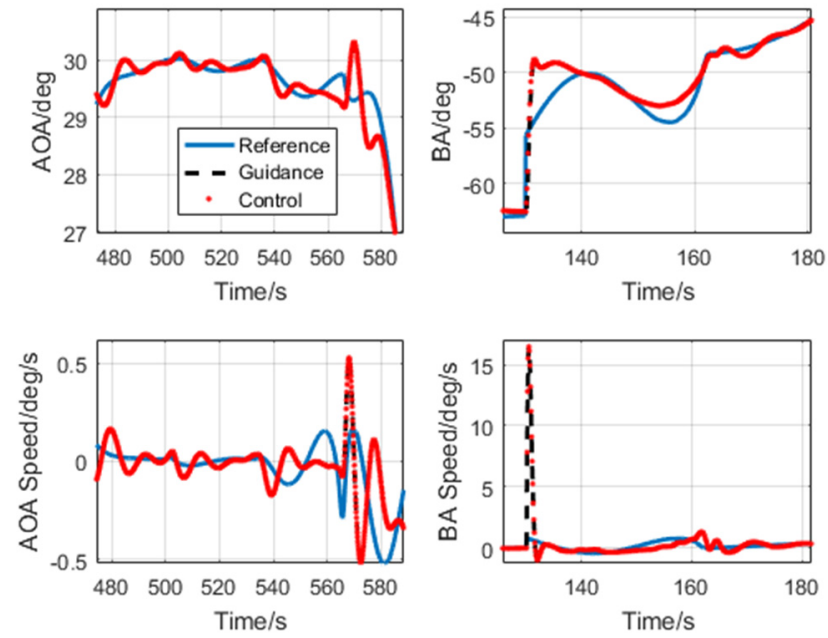

(b)

Figure 9. (a) Comparisons of $\mathrm{AOA}$ and BA provided by guidance system, control system and reference trajectory. (b) Enlarged comparisons of AOA and BA provided by guidance system, control system and reference trajectory.

\section{Conclusions}

In this paper, a novel IGC scheme for RHV is proposed with the capabilities of online aerodynamic coefficient estimation based on UKF and online trajectory generation based on the GPM. The LQR was adopted for trajectory tracking guidance and a second-layer SMC was designed for attitude control. The variation of lift and drag coefficients were modeled and estimated online, and, based on aerodynamic coefficient online estimation, a new trajectory can be generated online. The commands are actuator moments and their extremums impose more constraints on the rate and acceleration of flow angles. Comprehensive simulations were conducted. The final point position errors were within $120 \mathrm{~m}$ while velocity error is within $0.4 \mathrm{~m} / \mathrm{s}$ under normal conditions. With the novel IGC scheme proposed in this paper, the final point position errors were within $200 \mathrm{~m}$ while the velocity error was within $0.5 \mathrm{~m} / \mathrm{s}$ under large aerodynamic coefficient errors according to online generated trajectory. Comparable IGC performances with normal conditions prove the effectiveness and advantages of the proposed IGC scheme. 
Author Contributions: X.W., Y.L., and J.Z. conceived the presented ideas on the conceptualization, methodology and investigation. Writing-review and editing were done by X.W. and Y.L. Funding acquisition was done by X.W. All authors have read and agreed to the published version of the manuscript.

Funding: The research is funded by the Beijing Natural Science Funds [4204103] and Aeronautical Science Fund [2019ZC051009].

Institutional Review Board Statement: Not applicable.

Informed Consent Statement: Not applicable.

Data Availability Statement: The data is not available.

Conflicts of Interest: The authors declare that there is no conflict of interests regarding the publication of this article.

\section{References}

1. Zhang, W.; Chen, W.; Yu, W. Entry guidance for high-L/D hypersonic vehicle based on drag-vs-energy profile. ISA Trans. 2018, 83, 176-188. [CrossRef] [PubMed]

2. Shao, X.; Wang, H. Active disturbance rejection based trajectory linearization control for hypersonic reentry vehicle with bounded uncertainties. ISA Trans. 2015, 54, 27-38. [CrossRef] [PubMed]

3. Wang, X.; Zhang, J.; Wang, W.; Gao, P. An Innovative Architecture of UTC GPS/INS System with Improved Performance under Severe Jamming. Discret. Dyn. Nat. Soc. 2014, 2014, 185618. [CrossRef]

4. Wang, X.; Li, K.; Gao, P.; Wang, W. Reinforced Ultra-Tightly Coupled GPS/INS System for Challenging Environment. Math. Probl. Eng. 2014, 2014, 609154. [CrossRef]

5. Zhang, H.; Wang, H.; Li, N.; Yu, Y.; Su, Z.; Liu, Y. Time-optimal memetic whale optimization algorithm for hypersonic vehicle reentry trajectory optimization with no-fly zones. Neural Comput. Appl. 2020, 32, 2735-2749. [CrossRef]

6. Mao, Y.; Zhang, D.; Wang, L. Reentry trajectory optimization for hypersonic vehicle based on improved Gauss pseudo-spectral method. Soft Comput. 2017, 21, 4583-4592. [CrossRef]

7. Shao, X.; Wang, H.; Zhang, H. Enhanced trajectory linearization control based advanced guidance and control for hypersonic reentry vehicle with multiple disturbances. Aerosp. Sci. Technol. 2015, 46, 523-536. [CrossRef]

8. Schierman, J.D.; Hull, J.R.; Ward, D.G. On-Line Trajectory Command Reshaping For Reusable Launch Vehicles. In Proceedings of the AIAA GNC-Conference, Austin, TX, USA, 11-14 August 2003; pp. 2003-5439.

9. Qiao, H.; Sun, P.; Li, X. General Reentry Trajectory Planning Method Based on Improved Maneuver Coefficient. IEEE Access 2019, 7, 5447-5456.

10. Chai, R.; Xia, Y. Real-Time Reentry Trajectory Planning of Hypersonic Vehicles: A Two-Step Strategy Incorporating Fuzzy Multiobjective Transcription and Deep Neural Network. IEEE Trans. Ind. Electron. 2020, 67, 6904-6915. [CrossRef]

11. Tian, B.; Fan, W.; Zong, Q. Integrated guidance and control for reusable launch vehicle in reentry phase. Nonlinear Dynam. 2015, 80, 397-412. [CrossRef]

12. Wang, X.; Zhang, Y.; Liu, D.; He, M. Three-dimensional cooperative guidance and control law for multiple reentry missiles with time-varying velocities. Aerosp. Sci. Technol. 2018, 80, 127-143. [CrossRef]

13. Li, G.; Chao, T.; Wang, S.; Yang, M. Integrated Guidance and Control for the Fixed-trim Vehicle against the Maneuvering Target. Int. J. Control Autom. 2020, 18, 1518-1529. [CrossRef]

14. Yan, H.; Tan, S.; He, Y. A small-gain method for integrated guidance and control in terminal phase of reentry. ACTA Astronaut. 2017, 132, 282-292. [CrossRef]

15. Tian, B.; Zong, Q.; Wang, J.; Wang, F. Quasi-continuous high-order sliding mode controller design for reusable launch vehicles in reentry phase. Aerosp. Sci. Technol. 2013, 28, 198-207. [CrossRef]

16. Shao, X.; Wang, H. Back-stepping active disturbance rejection control design for integrated missile guidance and control system via reduced-order ESO. ISA Trans. 2015, 57, 10-22.

17. Mao, Q.; Dou, L.; Yang, Z.; Tian, B. Fuzzy Disturbance Observer-Based Adaptive Sliding Mode Control for Reusable Launch Vehicles With Aeroservoelastic Characteristic. IEEE Trans. Ind. Inform. 2020, 16, 1214-1223. [CrossRef]

18. Zang, L.; Lin, D.; Chen, S.; Wang, H.; Ji, Y. An on-line guidance algorithm for high L/D hypersonic reentry vehicles. Aerosp. Sci. Technol. 2019, 89, 150-162. [CrossRef]

19. Liu, K.; Hou, Z.; She, Z.; Guo, J. Reentry Attitude Tracking Control for Hypersonic Vehicle with Reaction Control Systems via Improved Model Predictive Control Approach. CMES Comput. Model. Eng. Sci. 2020, 122, 131-148. [CrossRef]

20. Seo, G.G.; Kim, Y.; Saderla, S. Kalman-filter based online system identification of fixed-wing aircraft in upset condition. Aerosp. Sci. Technol. 2019, 89, 307-317. [CrossRef]

21. Ferreres, G.; Hardier, G. Adaptive LFT control of a civil aircraft with online frequency-domain parameter estimation. Int. J. Robust Nonlinear 2019, 29, 5356-5376. [CrossRef] 
22. Subrahmanyam, S.; Kim, Y.; Ghosh, A.K. Online system identification of mini cropped delta UAVs using flight test methods. Aerosp. Sci. Technol. 2018, 80, 337-353.

23. Chowdhary, G.; Jategaonkar, R. Aerodynamic parameter estimation from flight data applying extended and unscented Kalman filter. Aerosp. Sci. Technol. 2010, 14, 106-117. [CrossRef]

24. Zhu, J.; Liu, L.; Tang, G.; Bao, W. Robust adaptive gliding guidance for hypersonic vehicles. Proc. Inst. Mech. Eng. Part G J. Aerosp. Eng. 2018, 232, 1272-1282. [CrossRef]

25. Garcia-Velo, J.; Walker, B.K. Aerodynamic Parameter Estimation for High-Performance Aircraft Using Extended Kalman Filtering. J. Guid. Control Dynam. 1997, 20, 1257-1259. [CrossRef]

26. Jorris, T.R. Common Aero Vehicle Autonomous Reentry Trajectory Optimization Satisfying Waypoint and No-Fly Zone Constraints; Air Force Institute of Technology: Wright-Patterson, OH, USA, 2017; pp. 88-94. 